\section{Factores de riesgo de ocurrencia y gravedad de malformaciones congénitas}

\author{
ANDREA CANALS C. ${ }^{1, \mathrm{a}}$, GABRIEL CAVADA C. ${ }^{1,2, \mathrm{~b}}$, JULIO NAZER H. ${ }^{3}$
}

\section{Identification of risk factors for congenital malformations}

\begin{abstract}
Background: The relative importance of congenital malformations as a cause of death in the first year of life is increasing along with the control of preventable causes of perinatal mortality. Aim: To identify risk factors for congenital malformations. Patients and Methods: Retrospective case-control study of births registered in the database of The Latin American Collaborative Study of Congenital Malformations (ECLAMC), in the period 2001-2010. Results: Birth weight and gestational age were significantly lower in cases than controls, behaving as risk factors and associated with a greater severity of congenital malformations. The risk and severity of congenital malformations increased along with mother's age. Fetal growth retardation, a history of congenital malformations in the family, physical factors and acute illnesses of the mother in the first trimester of pregnancy were also significant risk factors for congenital malformations and their severity. The educational level of the mother was a protective factor for congenital malformations and their severity. Conclusions: Variables previously identified as risk factors for congenital malformations, were significantly related with the occurrence of congenital malformations and their severity.
\end{abstract}

(Rev Med Chile 2014; 142: 1431-1439)

Key words: Congenital abnormalities; Risk factors.

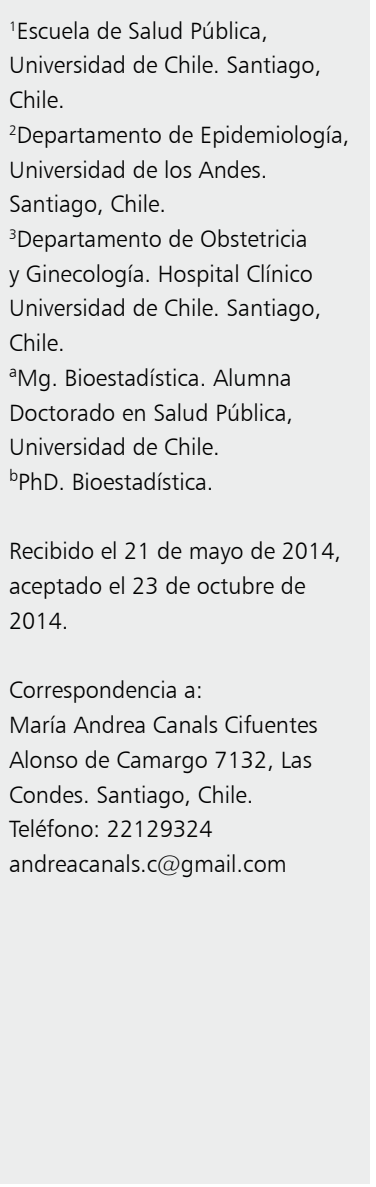

L as malformaciones congénitas (MFC) son alteraciones estructurales presentes al momento del nacimiento. El 50\% pueden prevenirse ${ }^{1-3}$ y el resto es de causa desconocida y en este punto la investigación es fundamental para mejorar la prevención.

En 1970, la mortalidad infantil (MI) en Chile era de 80 recién nacidos (RN) por mil nacidos vivos (NV), y la MI por MFC, era de 3,5 por mil, es decir, $4,4 \%$ de los niños que morían antes del año de edad, tenían como causa de muerte una o más MFC ${ }^{4}$. Gracias a las políticas implementadas por el Ministerio de Salud, patologías que tenían una alta mortalidad, como enfermedades infecciosas, respiratorias y desnutrición infantil, han sido controladas, con lo que la mortalidad infantil ha caído en forma progresiva hasta llegar a 7,9 por mil nacidos vivos en el $2009^{5}$. Sin embargo, la mortalidad por MFC se ha mantenido relativamente estable (2,9 por mil NV en 2009), lo que ha significado que la importancia relativa de ellas ha aumentado en forma importante, alcanzando $36,7 \%$ de las defunciones infantiles en $2009^{5}$.

Para reducir la mortalidad por MFC es necesario disminuir su prevalencia al nacimiento y existen dos maneras de hacerlo; a través del diagnóstico prenatal precoz del RN con MFC, de modo que sea recibido en un hospital con tecnología y personal capacitado para corregir o paliar su defecto y así mejorar su sobrevida, y en segundo lugar, hacer prevención primaria, es decir, evitar que se geste un niño con MFC. Para este segundo punto es de gran importancia la investigación de modo de identificar factores de riesgo de MFC.

Distintas medidas han logrado un avance en la detección de las MFC y en su prevención. La 
incorporación de la ecografía a fines de la década 1970-79, logró una mayor y más temprana detección de las malformaciones ${ }^{6}$. La campaña de vacunación masiva contra la rubeola logró prácticamente erradicar esta enfermedad, que al ser contraída por una mujer embarazada puede infectar al embrión provocando el síndrome de rubeola congénita ${ }^{7}$. También el control de embarazadas y medidas especiales en enfermedades crónicas como diabetes y epilepsia han ayudado a prevenir la ocurrencia de MFC.

El programa de prevención de los defectos de cierre del tubo neural mediante la fortificación de la harina con ácido fólico ha tenido un impacto importante en la prevalencia de MFC, logrando reducir en $51 \%$ la prevalencia al nacimiento de espina bífida y $42 \%$ de anencefalia ${ }^{8-10}$.

Diversas variables se han encontrado relacionadas con las MFC; edad de los padres, número de embarazos de la madre, espaciamiento entre ellos, etnicidad de los padres, patologías maternas y presencia de factores químicos, como algunos medicamentos usados en el primer trimestre del embarazo $^{11-12}$.

Los consumos de alcohol, drogas y tabaco por las madres han demostrado ser factores de riesgo de $\mathrm{MFC}^{13,15}$. Diversas patologías crónicas maternas como diabetes mellitus, hipertensión arterial, hipotiroidismo y asma bronquial, se han encontrado relacionadas con un mayor riesgo de $\mathrm{MFC}^{16-18}$.

Otros factores, como la restricción de crecimiento intrauterino (RCIU $)^{19}$, exposición a pesticidas, arsénico, etc., también se han encontrado relacionados con las $\mathrm{MFC}^{20-25}$.

El objetivo de este estudio es identificar factores de riesgo de MFC y comparar el riesgo de ocurrencia de MFC según gravedad, dados estos factores.

\section{Material y Método}

Se estudió la base de datos de recién nacidos en Chile entre 2001 y 2010, generada por el Estudio Colaborativo Latino Americano de Malformaciones Congénitas (ECLAMC). Este estudio registra, en cada hospital asociado a él, a todo RN, vivo o mortinato, que pese 500 gramos o más, que presente una o más MFC, mayores o menores. Para cada RN con MFC se registra como control al próximo $\mathrm{RN}$ sano del mismo $\mathrm{sexo}^{29}$.

Se registraron: peso de nacimiento, sexo, edad gestacional, edad materna, edad paterna, consumo de alcohol, tabaco y drogas en la madre, exposición a factores físicos (RX, traumatismos, otras radiaciones) y químicos (medicamentos) en el primer trimestre del embarazo, enfermedades agudas de la madre en el primer trimestre de embarazo, enfermedades crónicas, presencia de RCIU, nivel educacional de los padres, antecedentes étnicos, antecedentes de MFC en la familia, consanguinidad, y gravidez de la madre (número de embarazos).

Se estudió la normalidad de las variables mediante el test de Shapiro-Wilk, y la asociación entre la presencia de MFC y cada una de las variables restantes mediante los tests de Wilcoxon MannWhitney y $\chi^{2}$. Se calcularon Odds Ratio (OR) y se ajustaron modelos de regresión logística univariados y multivariados.

Se creó una variable que describe el estado de gravedad del RN. Esta se categorizó en 5 grupos; niños sanos, niños con MFC compatibles con la vida y susceptibles a corrección quirúrgica, niños con MFC compatible con la vida no susceptibles a corrección quirúrgica, niños con MFC incompatible con la vida y susceptibles a corrección quirúrgica, y niños con MFC incompatible con la vida y no susceptible a corrección quirúrgica. En caso de que un $\mathrm{RN}$ presentara más de una malformación registrada, correspondientes a distintas categorías de gravedad, se le asignó la categoría más desfavorable. Por ejemplo, si un RN presentaba dos MFC compatibles con la vida, siendo una corregible y la otra no corregible, se le asignó la categoría "MFC compatible con la vida, no corregible", y si un RN tenía tanto MFC incompatibles con la vida, corregibles como no corregibles, se le asignó la categoría "MFC incompatible con la vida, no corregible".

Se estudió la asociación de cada variable con la nueva variable respuesta, mediante los tests de Kruskal-Wallis y $\chi^{2}$. Se ajustaron modelos logísticos ordinales univariados y multivariados.

Los datos se procesaron en STATA 12, y se consideró una significación de 0,05.

\section{Resultados}

Entre 2001 y 2010, 14 hospitales chilenos participaron en el ECLAMC, aportando $22.227 \mathrm{RN}$ : 11.211 con MFC y 11.016 sanos (Tabla1).

Se excluyeron los RN correspondientes a los 
Factores de riesgo de malformaciones congénitas - A. Canals et al

Tabla 1. Distribución de casos y controles por hospital de procedencia. ECLAMC, Chile, 2001-2010

\begin{tabular}{|c|c|c|c|}
\hline Hospital & Casos & Controles & Total \\
\hline H. Clínico U. de Chile J.J. Aguirre & 1.455 & 1.457 & 2.912 \\
\hline H. Base Regional de Valdivia & 175 & 175 & 350 \\
\hline H. Clínico Pontificia U. Católica de Chile & 139 & 139 & 278 \\
\hline H. Las Higueras & 43 & 0 & 43 \\
\hline H. Naval Almirante Nef & 75 & 1 & 76 \\
\hline Clínica Sanatorio Alemán & 26 & 26 & 52 \\
\hline H. Regional de Rancagua & 2.011 & 2.010 & 4.021 \\
\hline H. Dr. Ernesto Torres Galdames & 69 & 0 & 69 \\
\hline H. Base de Linares Carlos Ibáñez del Campo & 1.373 & 1.373 & 2.746 \\
\hline H. Base Puerto Montt & 954 & 952 & 1.906 \\
\hline H. Dr. Sótero Del Río & 1.375 & 1.375 & 2.750 \\
\hline H. del Salvador & 403 & 403 & 806 \\
\hline H. San Borja Arriarán & 747 & 746 & 1.493 \\
\hline H. San José & 544 & 543 & 1.087 \\
\hline H. de Talca & 1.189 & 1.189 & 2.378 \\
\hline H. de Cauquenes & 157 & 157 & 314 \\
\hline H. de Curicó & 476 & 470 & 946 \\
\hline Total & 11.211 & 11.016 & 22.227 \\
\hline
\end{tabular}

hospitales Las Higueras de Talcahuano, Almirante Nef de Viña del Mar y Dr. Ernesto Torres Galdámez de Iquique debido al bajo número de controles. La muestra de estudio incluyó a 22.039 $\mathrm{RN} ; 11.024$ casos y 11.015 controles.

Se encontraron diferencias significativas entre casos y controles en todas las variables continuas estudiadas (Tabla 2). Se observaron menores pesos de nacimiento y edades gestacionales en casos que en controles, mientras que la edad paterna y materna fueron mayores en casos que en controles.
Se observó una relación significativa entre ocurrencia de MFC y escolaridad materna, paterna, antecedentes de MFC en la familia, consanguinidad, enfermedades agudas y crónicas, factores físicos, consumo de tabaco y RCIU (Tabla 3 ).

Mediante el análisis de modelos de regresión logística se llegó a un modelo final que incluye las variables edad gestacional, peso de nacimiento, RCIU, MFC en la familia, factores físicos, enfermedades agudas en el primer trimestre de embarazo y edad materna (Tabla 4, Figura 1).

Tabla 2. Resumen de variables continuas en casos y controles. ECLAMC, Chile, 2001-2010

\begin{tabular}{|lcccc|}
\hline Variable & $\begin{array}{c}\text { Casos } \\
\text { Promedio } \pm \text { d. estándar }\end{array}$ & $\begin{array}{c}\text { Controles } \\
\text { Promedio } \pm \text { d. estándar }\end{array}$ & OR (IC 95\%) \\
\hline Peso (gramos) & $3.155,2 \pm 757,9$ & $3.378,6 \pm 539,7$ & 0,99 & $(0,99-0,99)$ \\
Edad gestacional (semanas) & $38,05 \pm 3,0$ & $38,8 \pm 2,3$ & 0,90 & $(0,89-0,91)$ \\
Edad materna & $27,2 \pm 7,4$ & $26,1 \pm 7,0$ & 1,02 & $(1,02-1,03)$ \\
Edad paterna & $30,4 \pm 8,2$ & $29,5 \pm 8,0$ & 1,01 & $(1,01-1,02)$ \\
\hline
\end{tabular}


Tabla 3. Distribución de variables categóricas significativas en casos y controles, y OR respectivo. ECLAMC, Chile, 2001-2010

\begin{tabular}{|llrrr|}
\hline Variable & Categorías & Casos & Controles & OR (IC 95\%) \\
Gravidez & 1 = Un embarazo & $39,12 \%$ & $47,74 \%$ & - \\
& $2=2$ a 3 embarazos & $44,90 \%$ & $44,06 \%$ & $1,11(1,05-1,18)$ \\
& $3=4$ a 6 embarazos & $14,66 \%$ & $12,36 \%$ & $1,30(1,05-1,41)$ \\
& $4=7$ a 9 embarazos & $1,16 \%$ & $0,76 \%$ & $1,67(1,26-2,22)$ \\
Escolaridad materna & $5=$ Más de 9 embarazos & $0,15 \%$ & $0,07 \%$ & $2,25(0,97-5,22)$ \\
(B=básica, M=media, & 1 = Ninguna, B. incomp. & $12,66 \%$ & $11,39 \%$ & - \\
U=universitaria) & $2=$ B. comp. M. incomp. & $38,93 \%$ & $43,58 \%$ & $0,80(0,73-0,88)$ \\
& $3=$ M. comp. U. incomp. & $43,78 \%$ & $41,84 \%$ & $0,94(0,86-1,03)$ \\
Escolaridad paterna & $4=$ U. completa & $4,62 \%$ & $3,19 \%$ & $1,30(1,11-1,52)$ \\
(B=básica, M=media, & 1 = Ninguna, B. incomp. & $11,18 \%$ & $9,75 \%$ & - \\
U=universitaria) & $2=$ B. comp. M. incomp. & $36,88 \%$ & $40,26 \%$ & $0,80(0,72-0,88)$ \\
& $3=$ M. comp. U. incomp. & $45,58 \%$ & $45,68 \%$ & $0,87(0,79-0,96)$ \\
Malformaciones en la familia & $4=$ U. completa & $6,37 \%$ & $4,31 \%$ & $1,29(1,11-1,49)$ \\
Consanguinidad & & $28,78 \%$ & $13,00 \%$ & $2,71(2,52-2,90)$ \\
Enfermedades agudas & & $1,09 \%$ & $0,81 \%$ & $1,34(1,02-1,78)$ \\
Enfermedades crónicas & & $45,06 \%$ & $37,85 \%$ & $1,35(1,28-1,42)$ \\
Factores físicos & & $19,85 \%$ & $16,95 \%$ & $1,21(1,13-1,30)$ \\
\hline
\end{tabular}

Tabla 4. Odds ratio e intervalos de confianza del $\mathbf{9 5 \%}$ para variables incluidas en el modelo logístico

\begin{tabular}{|lcc|}
\hline Variable & OR & IC OR 95\% \\
Edad gestacional & 0,94 & $(0,93-0,95)$ \\
Peso al nacer & 0,99 & $(0,99-0,99)$ \\
RCIU & 1,53 & $(1,38-1,71)$ \\
Malformaciones en la familia & 2,74 & $(2,54-2,95)$ \\
Factores físicos & 1,55 & $(1,38-1,75)$ \\
Enfermedades agudas & 1,20 & $(1,13-1,28)$ \\
Edad materna & 1,02 & $(1,01-1,02)$ \\
\hline
\end{tabular}

Este modelo indica que las variables edad gestacional $(\mathrm{OR}=0,94)$ y peso de nacimiento $(\mathrm{OR}=0,99)$ serían factores que reducen el riesgo de MFC, y RCIU (OR = 1,53), MFC en la familia $(\mathrm{OR}=2,74)$, factores físicos $(\mathrm{OR}=1,55)$, enfermedades agudas $(\mathrm{OR}=1,20)$ y edad materna $(\mathrm{OR}=1,02)$, serían factores de riesgo.

$\mathrm{Al}$ categorizar los $\mathrm{RN}$ según gravedad de las MFC, se observó que las variables peso y edad gestacional serían factores que reducen el riesgo de gravedad de la malformación. Edad materna y paterna serían factores de riesgo de un cuadro más grave en el RN (Tabla 5).

Las variables categóricas que resultaron significativamente relacionadas con una mayor gravedad de las MFC, fueron las enfermedades agudas, enfermedades crónicas, factores físicos, factores químicos, consumo de tabaco, presencia de RCIU y gravidez de la madre. La escolaridad materna y paterna básica completa y media completa serían factores que reducen el riesgo de gravedad del RN respecto de la escolaridad básica incompleta. Una escolaridad universitaria completa sería factor de riesgo de mayor gravedad (Tabla 6).

Se ajustaron modelos logísticos ordinales multivariados para predecir la categoría de MFC. Se obtuvo un modelo final que incluye edad gestacional, peso de nacimiento, presencia de RCIU, MFC en la familia, factores físicos, enfermedades agudas en el primer trimestre de embarazo, edad y escolaridad materna (Tabla 7, Figura 2).

Para el modelo final se juntaron las categorías "educación media completa, universitaria incompleta" y "universitaria completa" de la variable 
Factores de riesgo de malformaciones congénitas - A. Canals et al

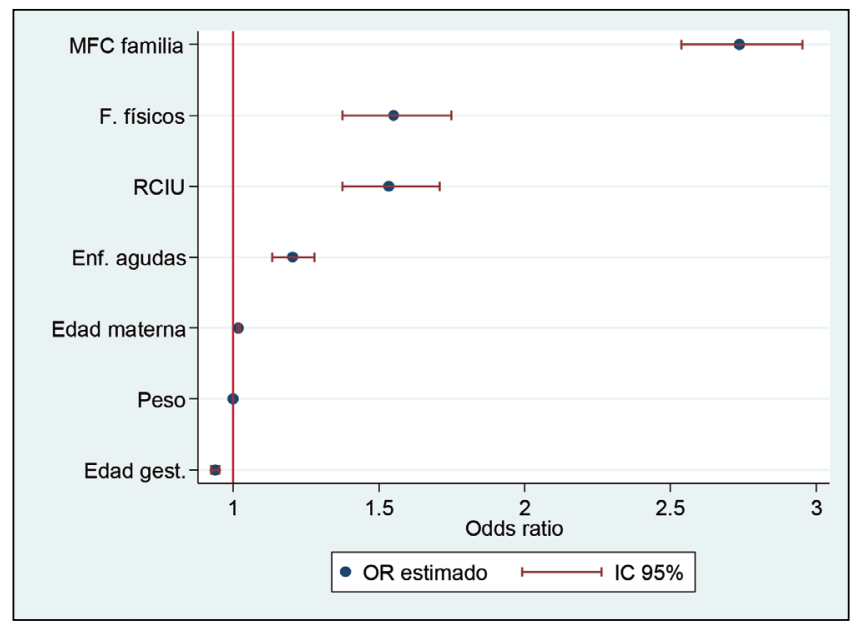

Figura 1. Odds ratio e intervalo de confianza del 95\% para variables incluidas en el modelo logístico multivariante. ECLAMC, Chile, 2001-2010.

Tabla 5. Resumen de variables continuas en recién nacidos sanos y con MFC según categoría, y OR según modelo logístico ordinal univariado. ECLAMC, Chile, 2001-2010

\begin{tabular}{|c|c|c|c|c|c|c|}
\hline Variable & $\begin{array}{l}\text { Sanos } \\
\text { Promedio } \pm \\
\text { d. estándar }\end{array}$ & $\begin{array}{c}\text { MFC } \\
\text { compatible } \\
\text { con la vida } \\
\text { corregible } \\
\text { Promedio } \pm \\
\text { d. estándar }\end{array}$ & $\begin{array}{c}\text { MFC } \\
\text { compatible } \\
\text { con la vida } \\
\text { no corregible } \\
\text { Promedio } \pm \\
\text { d. estándar }\end{array}$ & $\begin{array}{c}\text { MFC } \\
\text { incompatible } \\
\text { con la vida } \\
\text { corregible } \\
\text { Promedio } \pm \\
\text { d. estándar }\end{array}$ & $\begin{array}{c}\text { MFC } \\
\text { incompatible } \\
\text { con la vida } \\
\text { no corregible } \\
\text { Promedio } \pm \\
\text { d. estándar }\end{array}$ & $\begin{array}{c}\text { OR } \\
\text { (IC 95\%) }\end{array}$ \\
\hline Peso (g) & $3.155,2 \pm 757,9$ & $3.255,1 \pm 676,8$ & $3.127,2 \pm 757,4$ & $2.802,2 \pm 900,3$ & $2.253,2 \pm 883,0$ & $\begin{array}{c}0,99 \\
(0,99-0,99)\end{array}$ \\
\hline $\begin{array}{l}\text { Edad gestacional } \\
\text { (semanas) }\end{array}$ & $38,05 \pm 3,0$ & $38,37 \pm 2,74$ & $38,02 \pm 2,86$ & $36,85 \pm 3,51$ & $36,14 \pm 3,93$ & $\begin{array}{c}0,90 \\
(0,89-0,91)\end{array}$ \\
\hline Edad materna & $27,2 \pm 7,4$ & $26,46 \pm 7,01$ & $28,49 \pm 7,93$ & $27,92 \pm 7,50$ & $27,19 \pm 7,13$ & $\begin{array}{c}1,03 \\
(1,02-1,03)\end{array}$ \\
\hline Edad paterna & $30,4 \pm 8,2$ & $29,72 \pm 7,91$ & $31,51 \pm 8,63$ & $30,92 \pm 8,05$ & $30,67 \pm 7,75$ & $\begin{array}{c}1,02 \\
(1,01-1,02)\end{array}$ \\
\hline
\end{tabular}

escolaridad materna, al no encontrarse diferencias significativas entre las categorías.

Las variables RCIU, MFC en la familia, factores físicos, enfermedades agudas y edad materna serían factores de riesgo de ocurrencia de malformaciones de mayor gravedad en el RN. La edad gestacional, peso de nacimiento y escolaridad materna, serían factores que reducen el riesgo de la ocurrencia de malformaciones de mayor gravedad.

\section{Discusión}

El objetivo principal de este estudio era identificar factores de riesgo de MFC y de una mayor gravedad de estas mismas.
Se encontró que los valores de edad gestacional y peso de nacimiento son inferiores en casos que en controles, y en niños con MFC de mayor gravedad. Cabe preguntarse si esto significa que el bajo peso y la temprana edad gestacional son factores de riesgo y que corrigiéndolos evitaríamos el nacimiento de un niño malformado, o que más que factores de riesgo son una consecuencia de la malformación. Si este es el caso, serían más bien factores de sospecha que podrían sugerir la existencia de algún defecto estructural del feto o del RN. Las causas de bajo peso de nacimiento pueden ser la prematuridad y RCIU. Ambos se encuentran con mayor frecuencia en $\mathrm{RN}$ con $\mathrm{MFC}^{19}$, pero no son más que manifestaciones de sus patologías de base 


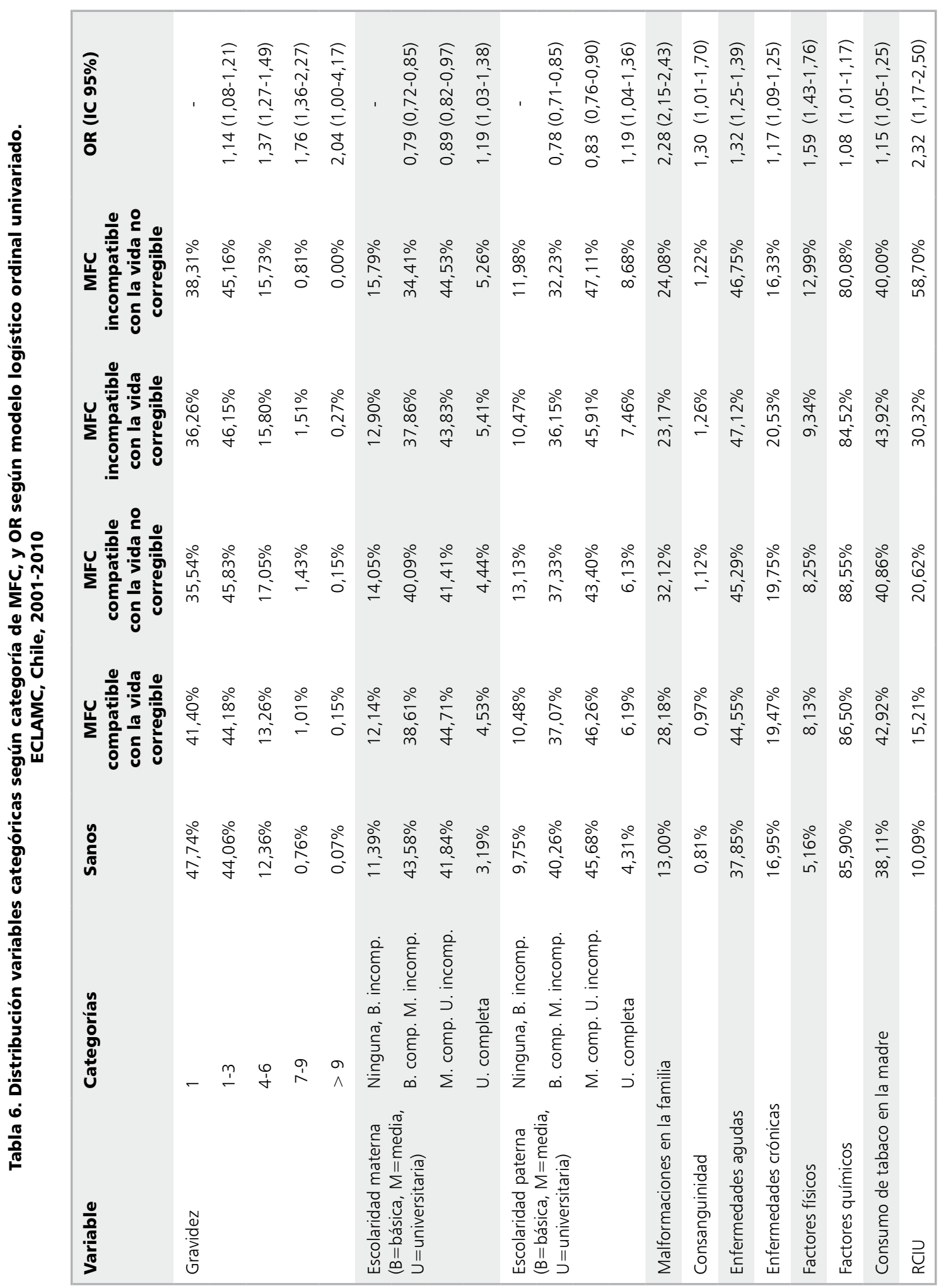


Factores de riesgo de malformaciones congénitas - A. Canals et al

Tabla 7. Odds ratio e intervalos de confianza para las variables incluidas en el modelo logístico ordinal multivariante

\begin{tabular}{|c|c|c|c|}
\hline Variable & OR & IC OR 95\% & Valor-p \\
\hline Edad gestacional & 0,95 & $(0,94-0,96)$ & $<0,0001$ \\
\hline Peso al nacer & 0,99 & $(0,99-0,99)$ & $<0,0001$ \\
\hline RCIU & 1,54 & $(1,40-1,70)$ & $<0,0001$ \\
\hline Malformaciones en la familia & 2,29 & $(2,14-2,44)$ & $<0,0001$ \\
\hline Factores físicos & 1,46 & $(1,32-1,63)$ & $<0,0001$ \\
\hline Enfermedades agudas & 1,17 & $(1,10-1,23)$ & $<0,0001$ \\
\hline Edad materna & 1,02 & $(1,02-1,03)$ & $<0,0001$ \\
\hline Ed. básica completa (materna) & 0,84 & $(0,76-0,91)$ & $<0,0001$ \\
\hline Ed. media completa (materna) & 0,90 & $(0,83-0,99)$ & 0,025 \\
\hline
\end{tabular}

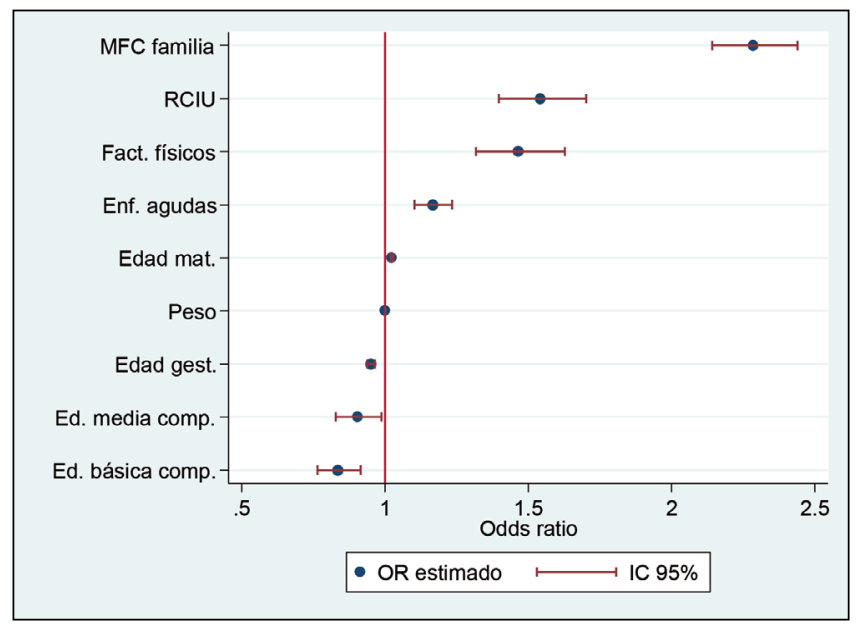

Figura 2. Odds ratio e intervalo de confianza del $95 \%$ para variables incluidas en el modelo logístico ordinal multivariante. ECLAMC, Chile, 2001-2010. y no origen de ellas. El hecho que ante un feto con RCIU tengamos más riesgo que el niño nazca con una malformación, no significa que la RCIU sea la causa de ella sino más bien una consecuencia.

Se ha demostrado que la edad materna es un factor de riesgo de MFC; a mayor edad aumenta el riesgo de tener un hijo con MFC, especialmente de origen cromosómico, como las trisomías 21, 13 y $18^{27-29}$. La edad del padre, si bien también es significativa como factor de riesgo, lo habitual es encontrar una relación directa de esta con la edad materna, siendo posible que su relación con la ocurrencia de MFC se deba a su relación con esta, ya que no está demostrado que la edad paterna sea por sí misma un factor de riesgo. De hecho, en este estudio, la relevancia de esta variable como predictora de la ocurrencia de MFC se pierde al ajustar por la edad materna.

Es conocido el riesgo de la consanguinidad como causa de transmisión de patologías de herencia autosómica recesiva. Sin embargo, esta variable se relacionó con un mayor riesgo de ocurrencia y de gravedad de MFC en los modelos univariados (Tabla 3, Tabla 6), pero pierde significación en los modelos multivariados (Tabla 4, Tabla 7).

La presencia de antecedentes de familiares con MFC es también un factor de riesgo, lo que se observó en este estudio con casi el triple de riesgo de ocurrencia de MFC en niños con antecedentes de MFC en la familia (Tabla 4) y aproximadamente el doble de riesgo de gravedad del RN (Tabla 7). Esta situación obedece a la presencia de genes en 
común en la familia, que pudieran ser responsables de anomalías de origen multifactorial o monogénicas (dominantes, recesivas, etc.), muchas de ellas con penetrancia incompleta y/o expresividad variable.

Es conocida también la relación entre las MFC y enfermedades agudas del primer trimestre del embarazo (Tabla 4, Tabla 7), especialmente las infectocontagiosas como la rubeola, y enfermedades crónicas como diabetes mellitus, hipertensión arterial e hipotiroidismo ${ }^{16-18}$. Es importante que el control obstétrico de la embarazada comience lo más precozmente posible, incluso desde antes del embarazo de modo de buscar estas patologías y tratarlas previamente.

También se ha descrito el carácter teratogénico de sustancias químicas contenidas en algunos medicamentos (factores químicos), como antiepilépticos, ácido retinoico contenido en cremas contra el acné, y otros que hay que evitar su consumo por las madres gestantes. Sin embargo, esta variable no resultó asociada a un mayor riesgo de MFC ni de gravedad del RN, al ajustar por el resto de las variables.

La exposición de la mujer a radiaciones, tanto dentales como de tórax y abdomen (factores físicos), también demostró ser un factor de riesgo importante (Tabla 4, Tabla 7). En general, al realizarse estos procedimientos se toman las medidas necesarias para evitar la exposición del embrión o feto, sin embargo, muchas veces las mujeres no saben que están embarazadas al someterse a estos exámenes. Es por esto que se deben extremar las precauciones al realizar este tipo de procedimientos en mujeres en edad fértil.

El nivel educacional de los padres se encontró relacionado con un mayor riesgo de malformaciones dada una mayor educación al comparar RN sanos y con MFC en el análisis bivariado, pero al ajustar por el resto de las variables en el análisis del modelo logístico binario, perdieron su efecto.

Sin embargo, al categorizar el grupo de RN con MFC según la gravedad de éstas, cambia el efecto de la escolaridad materna, demostrando ser un factor que reduce la gravedad de la MFC (Tabla 7). Se encontró que a mayor educación de la madre existe un menor riesgo de que el RN presente malformaciones graves. Esta relación posiblemente se deba a determinantes sociales que hacen que la mujer con mayor educación tenga un mejor nivel socio-económico y cultural que podría influir en un mayor acceso a la salud o hábitos de vida más saludables.

Ha sido descrita anteriormente la relación entre determinantes sociales tales como nivel socioeconómico y educación, y el nivel de salud de las personas. Un estudio analizó el riesgo de morir desde el nacimiento hasta los 2 años de edad en 13 países de América Latina en relación con la educación de la madre, encontrándose que el riesgo de morir del niño desciende a medida que la educación de la madre aumenta ${ }^{30,31}$.

Este estudio confirma, con un tamaño de muestra importante que incluye información de toda una década sobre $\mathrm{RN}$ en Chile, que ciertas variables no han cambiado su comportamiento como factores que reducen o aumentan el riesgo de MFC; exposición a factores físicos, edad materna, RCIU, enfermedades agudas de la madre en el primer trimestre de embarazo, y antecedentes de MFC en la familia. Sin embargo, la relación entre estas variables y la gravedad de las malformaciones es un aspecto novedoso que no había sido estudiado con esta metodología y demuestra que los factores de riesgo encontrados son además factores de riesgo para mayor gravedad de la MFC, lo que resalta aún más su importancia, pues son las anomalías más graves aquellas que más inciden en la morbimortalidad infantil. La relación encontrada entre el nivel educacional de la madre y un menor riesgo de malformaciones graves en el $\mathrm{RN}$ refleja la importancia de los determinantes sociales en la salud de las personas.

\section{Referencias}

1. Nazer J. Prevención primaria de los defectos congénitos. Rev Med Chile 2004; 132: 501-8.

2. Castilla EE, López-Camelo JS, Paz JE, Orioli IM. Prevención primaria de los defectos congénitos. Editorial Fiocruz, Río de Janeiro, Brasil, 2001.

3. Ten-Kate LP. Epidemiology of potentially avoidable birth defects. Eur. J. Epidemiol 1986; 2: 320-36.

4. Documento del Ministerio de Salud. División Programas de Salud. Programa de Salud del niño 1995.

5. Instituto Nacional de Estadísticas. Estadísticas Vitales. Informe anual 2009. [Internet]. Disponible en: http:// www.ine.cl/canales/chile_estadistico/demografia_y_vitales/estadisticas_vitales/2011/Vitales_2009_21102011. pdf.

6. Ortega D, Seguel S. Historia del ultrasonido: el caso chi- 
leno. Revista Chilena de Radiología 2004; 10 (2): 89-92.

7. Zapata L. Prevención y eliminación del síndrome de rubéola congénita. Revista de Obstetricia y Ginecología de Venezuela 2006; 66 (3): 193-6.

8. Castilla EE, Orioli IM, López-Camelo JS, Dutra MG, Nazer J. Preliminary data on changes in neural tube prevalence rates after folic acid fortification in South America. Am J Med Genet 2003; 123A: 123-8.

9. López-Camelo J, Orioli IM, Dutra MG, Nazer J, Rivera N, Ojeda ME, et al. Reduction of Birrth Prevalence Rates of Neural Tube defects after folic acid fortification in Chile. Am J of Medical genets 2005; 135: 120-5.

10. Nazer J, Cifuentes L. Resultados del Programa de Prevención de Defectos de Tubo Neural en Chile mediante la fortificación de la harina con ácido fólico. Período 2001-2010. Rev Med Chile 2013; 141: 751-7.

11. Cifuentes L, Nazer J, Catalán J, Parada L, Ruiz G. Malformaciones congénitas: Un modelo predictivo basado en factores de riesgo. Rev Med Chile 1989; 117: 611-7.

12. Nazer HJ, Cifuentes OL, Águila RA, Ureta LP, Bello MP, Correa CF, et al. Edad materna y malformaciones congénitas: Un registro de 35 años. 1970-2005. Rev Med Chile 2007; 135 (11): 1463-9.

13. Hackshaw A, Rodeck C, Boniface S. Maternal smoking in pregnancy and birth defects: a systematic review based on 173687 malformed cases and 11.7 million controls. Human Reproduction Update. 2011; 17 (5): 589-604.

14. Chung KC, Kowalski CP, Kim HM, Buchman SR. Maternal cigarrete smoking during pregnancy and the risk of having a child with cleft lip/palate. Plast Reconstr Surg 2000; 105 (2): 485-91.

15. Taboada Lugo N, León Mollinedo C, Martínez Chao S, Díaz Inufio O, Quintero Escobar K. Comportamiento de algunos factores de riesgo para malformaciones congénitas mayores en el municipio de Ranchuelo. Revista Cubana de Obstetricia y Ginecología 2006; 32 (2).

16. Nazer J, García Huidobro M, Cifuentes L. Malformaciones congénitas en hijos de madres con diabetes gestacional. Rev Med Chile 2005; 133: 547-54.

17. Nazer J, Ramírez R. [Congenital malformations in the offspring of diabetic mothers]. Rev Med Chile 2000; 128 (9): 1045-52.

18. Ordóñez MP, Nazer J, Águila A, Cifuentes L. Malformaciones congénitas y patología crónica de la madre. Estudio ECLAMC 1971-1999. Rev Med Chile 2003; 131 (4): 404-11.

19. Nazer J, Cifuentes L, Ramírez C, Seymour C, Águila A, Ureta $\mathrm{P}$, et al. Restricción del crecimiento intrauterino como factor de riesgo para malformaciones congénitas.
Revista Chilena de Obstetricia y Ginecología 2009; 74 (6): 366-71.

20. Wo J, Chen G, Liao Y, Song X, Pei L, Wang J, et al. Arsenic levels in the soil and risk of birth defects: A population-based case-control study using GIS technology. Journal of Environmental Health 2011; 74 (4): 20-5.

21. Benítez-Leite S, Macchi ML, Acosta M. Malformaciones congénitas asociadas a agrotóxicos. Rev Chil Pediatr 2009; 80 (4): 377-8.

22. Rojas A, Ojeda M E, Barraza X. [Congenital malformations and pesticide exposure]. Rev Med Chile 2000; 128 (4): 339-404.

23. Gaspari L, Sampaio DR, Paris F, Audran F, Orisini, M, Neto JB, Sultan C. High prevalence of micropenis in 2710 male newborns from an intensive-use pesticide area of Northeasthern Brazil. Int J Androl 2012; 35 (3): 253-64.

24. Brender JD, Werler MM, Kelley KE, Vuong AM, Shinde MU. Nitrosatable drug exposure during early pregnancy and neural tube defects in offspring: National Birth Defects Prevention Study. Am J Epidemiol 2011; 174 (11): 1286-95.

25. Snijder CA, Vlot IJ, Burdof A, Obermann-Borst SA, Helbing WA, Wildhagen MF, Steegers EA, SteegersTheunissen RP. Congenital heart defects and parental occupational exposure to chemicals. Hum Reprod 2012; 27 (5): 1510-7.

26. ECLAMC: Manual Operacional. 2002. Edición electrónica: http:/eclamc.fiocruz.br

27. Nazer J, Cifuentes L. Estudio epidemiológico global del síndrome de Down. Rev Chil Pediatr 2011; 82 (2): 10512.

28. Nazer J, Cifuentes L. Prevalencia de malformaciones congénitas en hijos de madres mayores de 34 años y adolescentes. Hospital Clínico de la Universidad de Chile, 2002-2011. Rev Chil Obstet Ginecol 2013; 78 (4): 298-303.

29. Pardo R, Nazer J, Ramírez C, Faundes V, Cifuentes L. Características epidemiológicas de los neonatos con cromosomopatías nacidos en el Hospital Clínico Universidad de Chile. Período 2001-2010. Rev Hosp Clin Univ Chile 2013; 24 (3): 181-7.

30. Álvarez L. Los determinantes sociales de la salud: más allá de los factores de riesgo. Revista Gerencia y Políticas de Salud 2009; 8 (17): 69-79.

31. Behm H. Determinantes económicos y sociales de la mortalidad en América Latina. Salud Colectiva 2011; 7 (2): 231-53. 